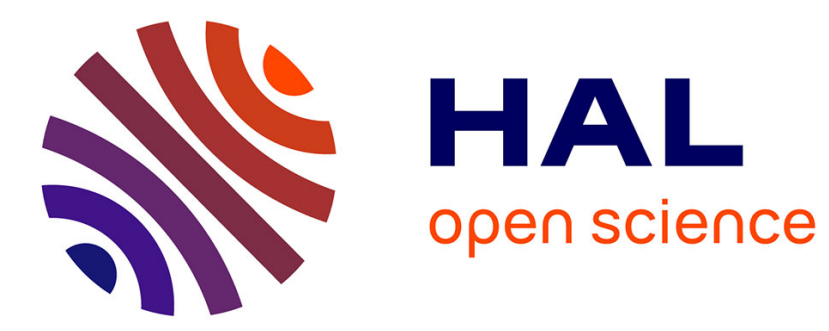

\title{
Le Care comme partie émergée de la production de la vie
} Daniel Bertaux

\section{- To cite this version:}

Daniel Bertaux. Le Care comme partie émergée de la production de la vie. Revue des Sciences sociales, 2014, Vers une société du CARE?, 52, pp.118-128. hal-01295091

\section{HAL Id: hal-01295091 \\ https://hal.science/hal-01295091}

Submitted on 30 Mar 2016

HAL is a multi-disciplinary open access archive for the deposit and dissemination of scientific research documents, whether they are published or not. The documents may come from teaching and research institutions in France or abroad, or from public or private research centers.
L'archive ouverte pluridisciplinaire HAL, est destinée au dépôt et à la diffusion de documents scientifiques de niveau recherche, publiés ou non, émanant des établissements d'enseignement et de recherche français ou étrangers, des laboratoires publics ou privés. 
REVUE DES

SGIENGES

SOMMAIRE

VERS UNE SOCIÉTÉ DU CARE? 2014 n52

PRÉSENTATION

p.4 BERNARD WOEHL, PHILPPE HAMMAN \& FREDDY RAPHAËL

p. 8 JUAN MATAS

p.80 CAROLINE GIACOMONI, HÉLEENE HOARAU

.12 DANIEL BERTAUX \& CATHERINE DEL CROIX

DES FLUX MIGRATOIRES DE TRAVAILLEUSES DU CARE

p. 18 HELMA WTZ \& EWA PALENGA-MÓLLENBECK divisée. Liens et transnationa

JUAN MATAS

(n)

D. 42 NATACHA BORGEAUD-GARCIANDA Relations de care, affects ef dominations. Le care à

FRANCE -ALLEMAGNE

ÉTUDES COMPARATIVES DE FORMES DE CARE

D.54 JANINA GLAESER

Assistmilesen methe et politiques de la petite entance : une comparaiso France-Allemagne

p.62 JULLE SENTIS

Apprendre les métiers du care en France et en Allemagne

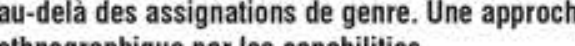

LE CARE EN INSTITUTION

TROIS ÉTUDES DE CAS EN FRANCE

p.72 MOUNRFARHAT

MOUNIR FARHAT personnel et distance professionnelle en unité Alzheimer

P. 80 CAROLINE GIACOMONI, HÉLEENE HOARAU
\& ALAIN MONNEREAU Enjeux et conflits autour du care à travers l'expérience de la délégation à domicile des traitents en concérologie

p. 86 LAURELINE COULOMB Léthique du care malmenée au cours des interactions entre infirmiers et individus sans-abri LUTTES ET RÉFORMES POTENTIELLES

p. 94 URSULA APITZSCH net l'ordre de genre

p. 102 AGNËS BERTHELOT-RAFFARD La discrimination par association : une expression du care dominé

p. 110 CHANTAL NICOLE-DRANCOUR Pour une reconnaissance du care dans des sociétés de pleine activité

p. 118 DANEL BERTAUXX

政 CHANTIERS DE RECHERCHE

p. 132 ÉLOOIE VALENTIN

Emotions, dynamiques citoyennes et espace public. L'expérience du projet social d'une maison de quartier a Dunkerque

p. 142 SUZY GUTH Conflit dans le vignoble : Ammerschwihr et l'AOC Kaefferkopf

LU - À LIRE

p. 154 Recensions

p. 166 Résumés des articles

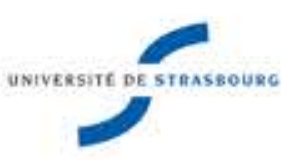

Cirs

Revve bénéficiant du soutien de l'Isstitut
des Sciences Humaines el Sociales du CNRS
SSN 1623-6572

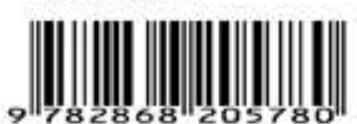

REVUE DES SOIENOES SOBALES

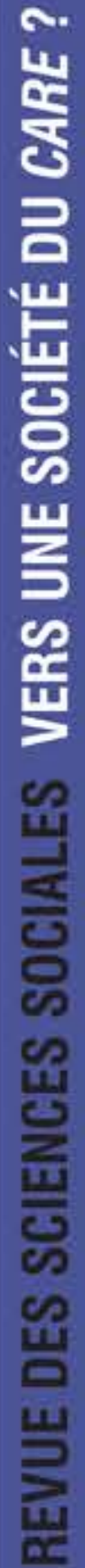

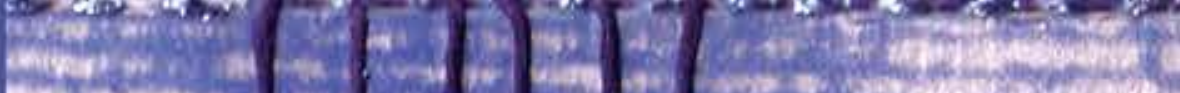

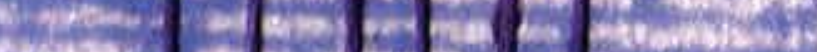

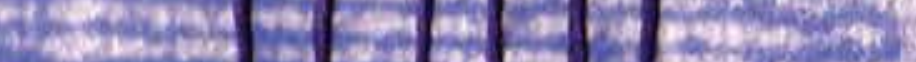
6.

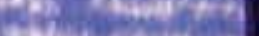
wast:

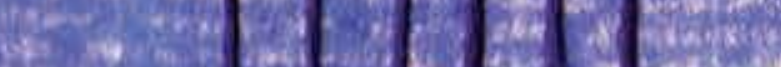

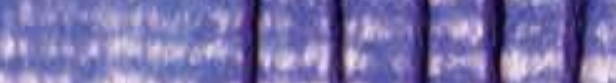

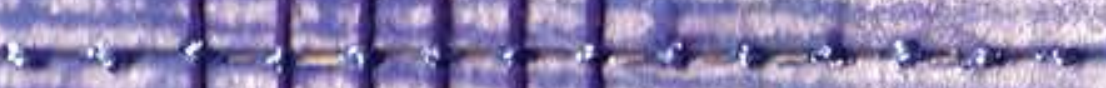
-

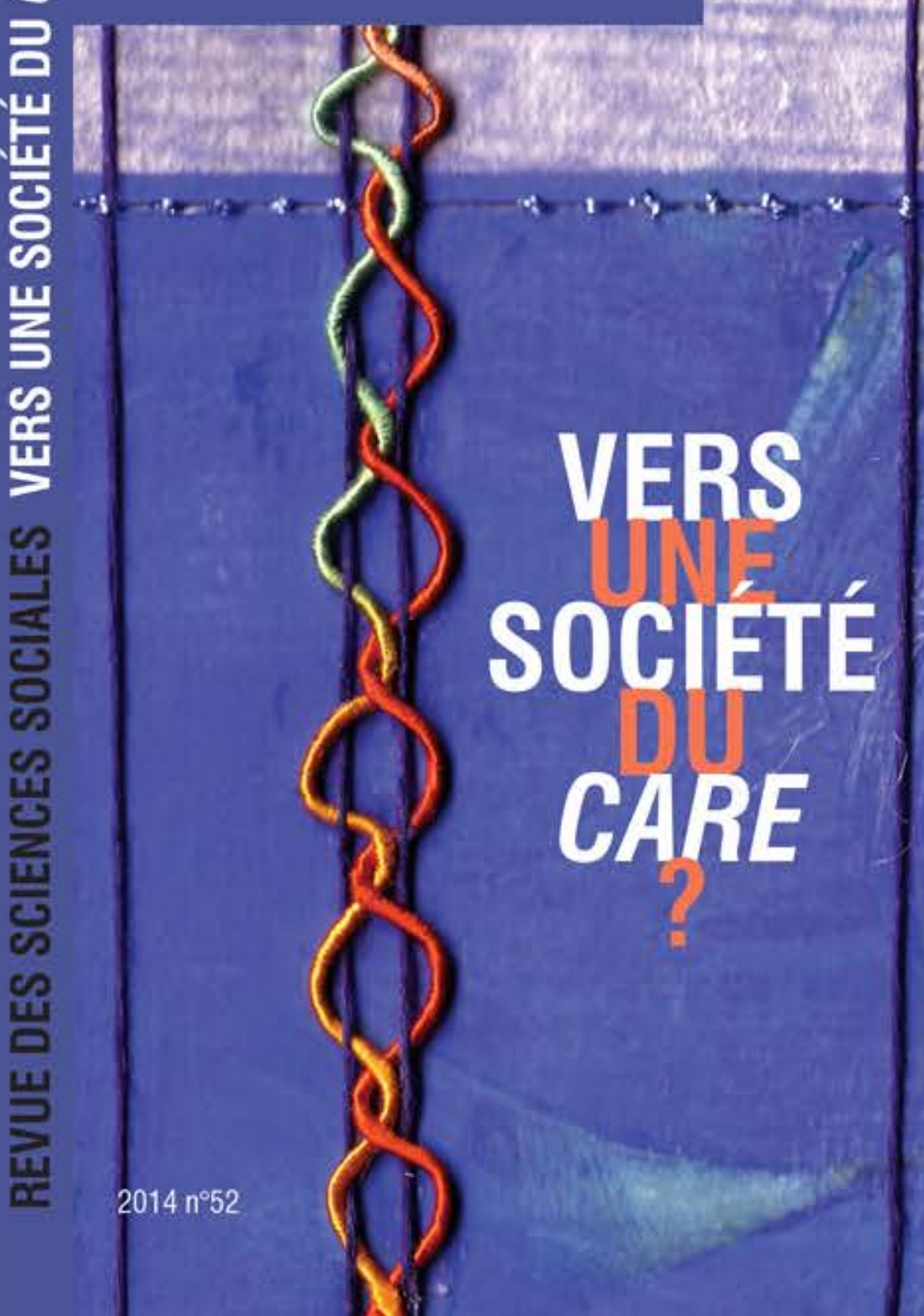


REVUE DES

SAIN

SOGALES

VERS UNE SOCIÉTÉ DU CARE ?

$2014 n^{\circ 5}$ ?

- Directeur scientifique

Freddy Raphaël

- Rédacteur en che

Patrick Ténoudji

- Comité scientifique

Gerges Balandier (EHESS Paris)

(CENRS Toulouse).

(MaymTP Paris) Gionas Paris), Jean Cuisenier

Milano). Jose Carios Gomes da Silva (Portugal).

François Hèran (INED Paris), Claude Javeau (Univ. Libre

de Bruxelles). Nicole Lapierre (EHESS/CNRS Paris).

Marianne Mesnil (Univ. Libre de Bruxelles).

Sonia Montecino (Univ. de Chile), Jean Rèmy (Univ.

Alain Tarrius (Univ. Toulouse-Le Mirail), Alain Touraine

(CEMS Paris)

- Comité de rédaction

Chelle Biannuis-Gasser (Univ. Tours), Maurice Blane Univ. Strasbourg), Nicoletta Diasio (Univ. Strasbourg). Woltong Strasbourg). Antida Gazzola (Univ. Genova). Philippe Hamman (Univ. Strasbourg). Pascal Hintermeyer (Univ. Strasbourg), Leila Jeolas (Uniy Londrina), Reinhard Johler (Univ, Túbingen). Salvatore La Mendola (Univ. Padova), David Le Breton (Univ. Strasbourg/UF), Juan Malas (Univ. Strasbourg. Gabriele Profita (Univ. Palerme), Ilario Rossi (Univ. Lausanne), Patrick Schmoll (CNRS Strasbourg), Roger Some (Uni. Slasbourg), Simona tersign

Collaborateurs éditoriaux

Anny Bloch, Marie-Noële Dents,

Anny Bloch, Marie--Noële Der
- Équipe iconographique sur ce numéro

Vincent Hans, Aline Mathy, Patrick Schmo

- Ce numero a été coordonné par :

Catherine Delcroix, Juan Matas \& Daniel Bertaux

- Maquette

Couverture : Aline Mathy

Presses Universitimires de Stroshour

- Administration

Presses Universitaires de Strasboutro

5 allée du Gal Rouvillois - CS 50008

FR-67083 Strasbourg Cedex

Tél. 0368856265

e-mail : pu-strasbourg@unistra.tr

- Diftusion/distribution

agences d'abonnement :

cid@msh-paris.tr

(nstitutions:

www.lcdpu.fi

18 rue Robert-Schuman

CS 90003

FR-94227 Charenton-le-Pont Ceder

Tel. 0153485630

Revue publiée par la Faculté des sciences sociale

de TUniversité de Strasbourg et le laboratioire

DST 1023.0572 (2)

www.revue-des-sciences-sociales.com
Ál'attention des auteurs

Les articles sont à adresser par document attachẻ à l'attention de Patrick Ténouditi, rédacteur en chêt <patrick.tenoudji@misha.fr>

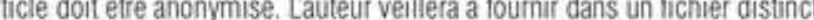

- sestitres ou protession et son appantenance instiutionnelle (université, centre de recherche)

- un résumé d'une dizaine de lignes en français et en anglais.

Le texte comporte au maximum 40000 signes et blancs, notes et bibliographie comprises. II est Myhme par des intertitres courts, avec deux niveaux dintertitres au maximum. Les passages cites de rextes en langues tlangertes sont traduns en lançais. Les nolts, oni

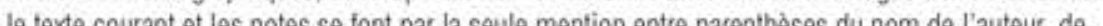
l'année de publication et, le cas échéant, des numéros de pages citées.

Exemples

- ... a ainsi que lindique J. Dupont (2003a) ...

-.... cette question a lairt objel de plusieurs travaux (Oupont 2003a, Durand 2004. 2007) ...

La bibliographie suit les consignes de présentations suivantes :

- pour un ouvage . Oupont J. (2003a), Thre de rouvrage en iltafique, Lieu d'édition, Editeur.

- pour un chapitse douvrage : Durand M. (2004). Titre du chapitre sans guillemets, in Dupont

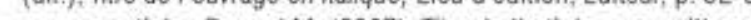

-

Les articles soumis par les auteurs sont examinés chacun par un comité d'au moins deux lecteurs qui rendent des avis séparés. Lexemplaire du texte qui leur est remis ne mentionne pas lidentité de Tauteur, et lauteur de son cote ne peut se laire communiquer T identitie de ses lecteurs : la decision

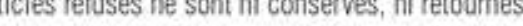

Si des graphiques et illustrations sont nécessaires à l'appui du texte, l'auteur en adresse les originaux ou les fichiers image haute résolution (300dpi) à la rédaction. Hors ce cas de figure, la recherche la rédaction de la revue. 


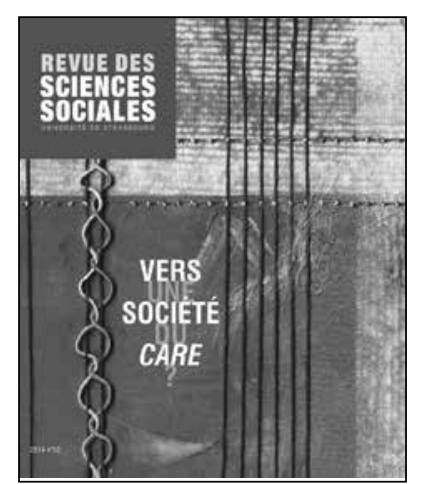

Khadija Seddiki

artiste plasticienne, diplômée de l'Académie royale des beaux-arts de Belgique, de l'Académie des arts et métiers

Constantin Meunier d'Etterbeek, des Écoles supérieures des beaux-arts d'Alger et d'Oran, et de l'Université Denis Diderot-Paris VII. Elle a fondé TISSART, école d'art et atelier de tissage, à Vanves. http://khadijaseddiki.com/
Dans les sociétés du "Nord" de la planète, les besoins de care augmentent alors que les femmes n'acceptent plus guère de s'y consacrer sans contrepartie. Ce déséquilibre engendre des flux importants et différenciés de migrantes venant de «l'Est» ou du «Sud». Qui sont-elles, dans quelles conditions viennent-elles, dans quels cadres, lieux, rapports et conditions concrètes travaillent-elles, quels sont leurs droits?

Ce numéro examine la situation de travailleuses du care: Polonaises en Allemagne, Péruviennes à Buenos Aires ou au Chili; il aborde les contextes institutionnels de ces activités en France, en Allemagne, dans une perspective comparative. Une "société du care» est-elle en train d'émerger?
Couverture

image: Khadija Seddiki composition: Aline Mathy 


\section{PRÉSENTATION}

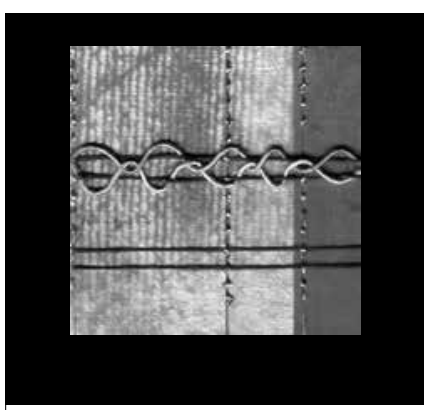

4

BERNARD WOEHL

PHILIPPE HAMMAN \& FREDDY RAPHAËL

Hommage

à François Steudler 8

JUAN MATAS

Introduction

12

DANIEL BERTAUX \& CATHERINE DELCROIX

Présentation
DES FLUX MIGRATOIRES DE TRAVAILLEUSES DU CARE

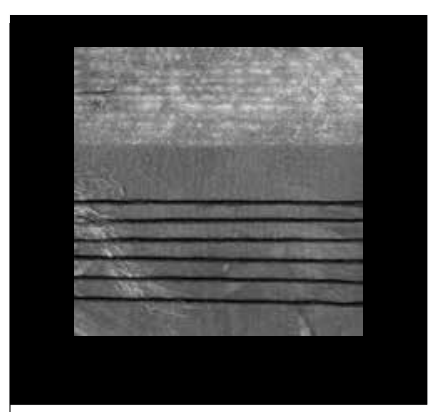

18

HELMA LUTZ \&

EWA PALENGA-

MÖLLENBECK

Les migrantes du care dans

l'Europe divisée. Liens et

contradictions dans un

espace transnational

\section{8}

\section{JUAN MATAS}

Les migrantes péruviennes au Chili et le travail de care

opportunités et risques

d'une situation en plein essor

\section{2}

NATACHA BORGEAUD-

\section{GARCIANDA}

Relations de care, affects et dominations. Le care à demeure à Buenos Aires
FRANGE -ALLEMAGNE : ÉTUDES COMPARATIVES DE FORMES DE CARE

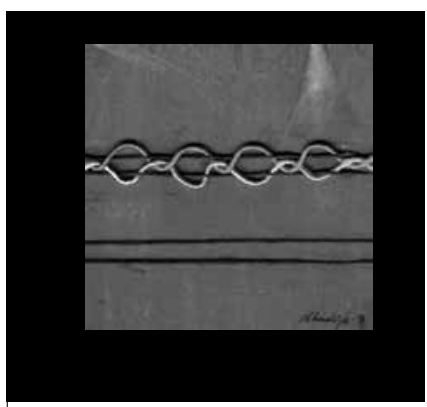

54

\section{JANINA GLAESER}

Assistant(e)s maternel(le)s d'origine étrangère et politiques de la petite enfance: une comparaison France-Allemagne

\section{2}

\section{JULIE SENTIS}

Apprendre les métiers du care en France et en Allemagne au-delà des assignations de genre. Une approche ethnographique par les capabilities
LE GARE EN INSTITUTION : TROIS ÉTUDES DE CAS EN FRANGE

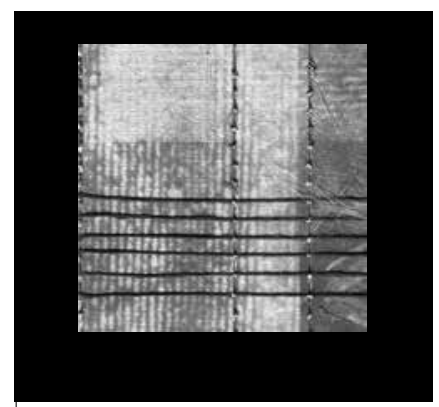

72

\section{MOUNIR FARHAT}

Un dilemme du care:

tension entre engagement personnel et distance professionnelle en unité Alzheimer

80

CAROLINE GIACOMONI, HÉLĖNE HOARAU \& ALAIN MONNEREAU

Enjeux et conflits

autour du care à travers

l'expérience de la délégation à domicile des traitements en cancérologie

86

\section{LAURELINE COULOMB}

L'éthique du care malmenée au cours des interactions entre infirmiers et individus sans-abri 


\section{LUTTES ET RÉFORMES POTENTIELLES}

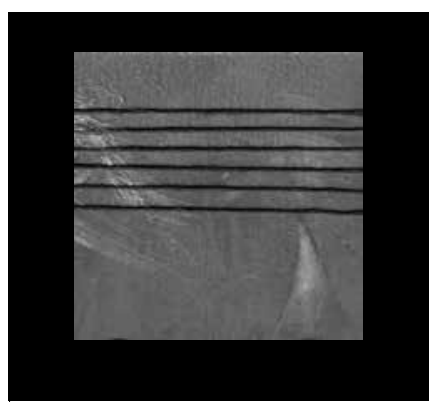

94

URSULA APITZSCH

Le care, la migration

et l'ordre de genre

102

AGNÈS BERTHELOT-RAFFARD

La discrimination

par association:

une expression

du care dominé

110

CHANTAL NICOLE-

\section{DRANCOURT}

Pour une reconnaissance

du care dans des sociétés

de pleine activité

\section{8}

\section{DANIEL BERTAUX}

Le care comme partie

émergée de la production

de la vie

\section{CHANTIERS} DE RECHERCHE

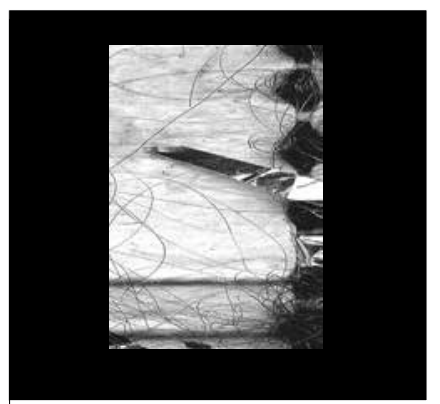

132

ELODIE VALENTIN

Émotions, dynamiques

citoyennes et espace public. L'expérience du projet social

d'une maison de quartier à

Dunkerque

\section{2}

\section{SUZY GUTH}

Conflit dans le vignoble:

Ammerschwihr

et I'AOC Kaefferkopf
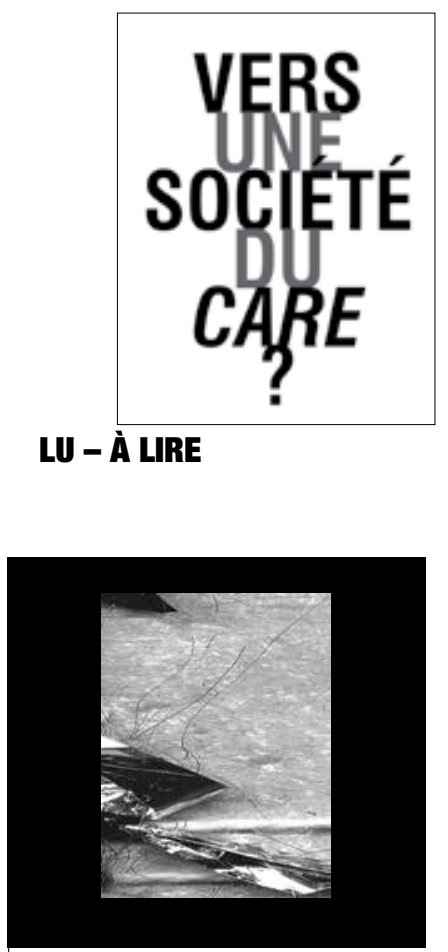

154

RECENSIONS

166

RÉSUMÉS DES ARTICLES 


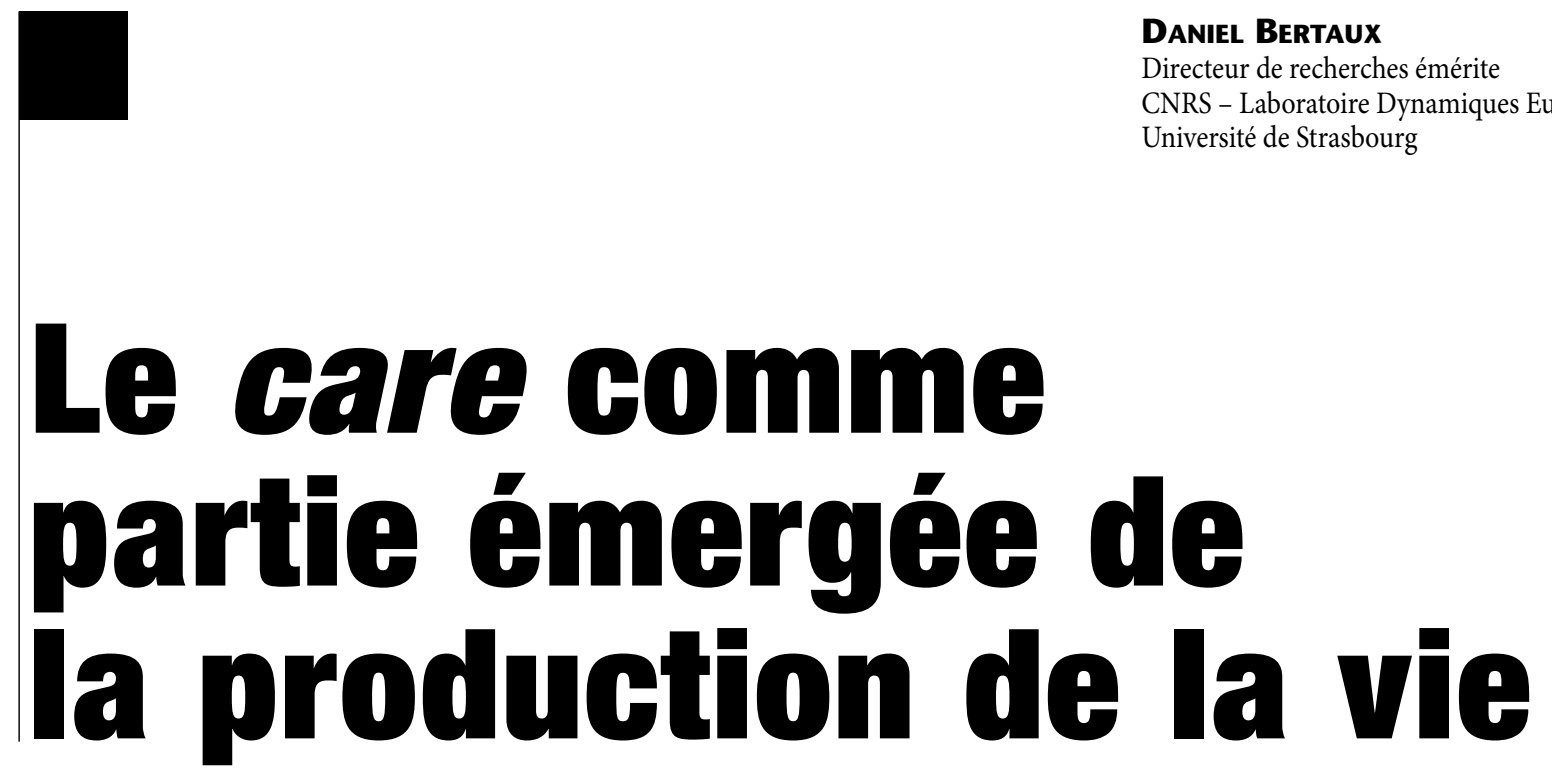

\section{Care et anthroponomie.}

Qu'entend-on par care ('soin direct') dans la littérature scientifique contemporaine, sinon un service qu'une personne (le carer, le 'soigneur') rend dans une interaction en face-à-face à une autre personne (le cared, le 'soigné') qui se trouve, temporairement ou de façon chronique, dans une situation objective de dépendance?

Le care y est donc défini par son contenu: celui de l'activité elle-même. Mais à elle seule cette caractéristique très singulière aurait dû attirer l'attention.

En sciences sociales il est en effet tout à fait exceptionnel qu'une activité soit pensée à travers son contenu - comme le fait la pensée spontanée - plutôt qu'à travers le type d'organisation, le type de rapports sociostructurels au sein desquels elle s'effectue. Or le care, comme la culture de tomates, la couture, la cuisine et bien d'autres activités, peut s'effectuer aussi bien dans le cadre familial-domestique que dans celui d'un rapport marchand simple (babysitter, aide à domicile...), celui d'une entreprise privée tendue vers le profit (maison de retraite haut-de-gamme), celui d'une entreprise publique (crèche publique, asile, maison de retraite de statut public), ou encore celui d'une association sans but lucratif; voire celui de relations d'échanges de services non-marchandisés entre voisin(e)s ou amies ${ }^{1}$.

L'analogie avec la cuisine, la couture, ou la culture de tomates (respectivement un service, une activité de production artisanale ou industrielle, une activité agricole) vise à rappeler la généralité du principe analytique selon lequel ce n'est pas le contenu en soi d'une activité donnée, mais le cadre de rapports sociostructurels au sein duquel elle est effectuée qui définit à la fois les positions respectives des agents/acteurs qui y sont impliqués, la répartition des tâches et des responsabilités entre eux, leurs motivations respectives et les significations qu'ils confèrent à leur activité, leurs modes de rétribution, bref l'économie à la fois matérielle et morale de l'ensemble ${ }^{2}$.

Pourtant l'objet care, trente-deux ans après sa naissance, semble refuser encore de se laisser penser comme n'importe quelle autre activité collective: à partir de tel ou tel type d'organisation, du type de cadre structurant cette activité. Et il semble aussi réti- cent à quitter l'improbable berceau qui l'a vu naître: celui de l'éthique.

Rien ne semblait en effet prédisposer l'étoile neuve du care à apparaître dans cette région de la pensée ${ }^{3}$. Car enfin, il s'agit d'un type particulier de service direct aux personnes, catégorie qui en contient d'autres (coiffure, soins esthétiques, coaching...; restauration, taxis...) et qui a sa place, aux côtés de maintes autres catégories, dans l'ensemble beaucoup plus vaste des Services: une méga-catégorie hétéroclite au sein de laquelle les statisticiens regroupent toutes sortes d'activités.

Enfin, troisième caractéristique surprenante, si depuis la parution de l'ouvrage séminal de Gilligan (1982; cf. aussi Gilligan 1977) se sont multipliées les études théoriques et empiriques sur telle ou telle caractéristique du care, les rapports entre ce type d'activité et d'autres types de services, sa place au sein d'un ensemble plus vaste d'activités n'ont guère fait l'objet de réflexions approfondies.

C'est précisément à ce dernier point que le présent article est consacré. On y conceptualisera les activités de care comme des activités de production: non pas certes de production d'objets tangibles, mais néanmoins de production (et de 're-produc- 
tion') de la vie dans les corps. On y montrera que le care ne constitue que l'une des formes - spécifique aux personnes les plus vulnérables - d'un ensemble beaucoup plus vaste d'activités de production des corps 'totaux' (physico-psychiques); ensemble qui comprend entre autres les activités de soins médicaux et les activités d'enseignement, deux secteurs extrêmement dynamiques et diversifiés. Enfin on développera la thèse selon laquelle, parce que ces grands secteurs d'activité (care, santé, enseignement...) ont pour finalité ultime de préserver la vie et les chances de vie, leur logique immanente résiste à l'emprise omniprésente du 'marché' (lire: du capital privé) et pousse, tout au contraire, à privilégier la forme publique pour leur financement, leur organisation, et même leur gestion quotidienne.

On touche ici au cour de la question. Le care n'est en fait que la partie émergée d'un ensemble beaucoup plus vaste et diversifié - encore invisible d'activités de services aux personnes qui constituent ainsi une 'seconde production' parallèle à la production des choses. Il ne s'agit donc pas seulement de reproduction, terme condescendant qui laisse entendre qu'il n'y a rien de dynamique ou d'innovant de ce côtélà; il s'agit bien d'une production, qui ne le cède en rien en ampleur et en complexité, en innovations, en dynamisme, à la production des choses (l'économie matérielle).

Cette 'seconde production' n'a guère encore été perçue - et encore moins pensée - comme telle. Comme l'économie matérielle jusqu'au milieu $\mathrm{du} \mathrm{XVIII}^{\mathrm{e}}$ siècle, elle navigue encore sous les eaux, hors de portée des regards conceptuels. Ou plus exactement, une petite partie vient d'en émerger récemment dans la pensée collective; et c'est, précisément, le care. Mais le care n'est rien de plus que le sommet du crâne de la baleine, sommet rendu enfin visible grâce à l'intuition de Carol Gilligan. Le présent article vise à esquisser les contours de cette baleine elle-même, c'est-à-dire de cet immense ensemble d'activités de production de la vie que nous appelons production anthroponomique .

\section{Une seconde production}

Dans les sociétés de la modernité la production 'des gens eux-mêmes' (la production anthroponomique, Bertaux 1977), bien loin de se réduire à de la reproduction, n'est 'seconde' que par rapport à la production que nous tenons encore pour 'première' : la production des choses. Cette seconde production comprend bien évidemment les activités domestiques (et par extension, familiales), y compris le care ayant pour objet les enfants voire d'autres personnes dépendantes, activités orientées à la fois par la nécessité et par des projets (projections sur les enfants, Nicole-Drancourt 1989) et y répondant selon une logique du don à la Mauss. Mais elle comprend aussi les formes institutionnalisées du care (ainsi les orphelinats, asiles psychiatriques, centres pour personnes âgées dépendantes) qui suivent d'autres logiques (marchande, publique, associative). Elle inclut également nombre d'autres activités de services aux personnes, proposées en général sous une forme marchande simple (artisanale ou profession libérale): restauration, coiffure et soins du corps, psychothérapie...

Mais ce sont surtout deux grands secteurs d'activité qui désormais figurent au centre de la seconde production: les soins médicaux d'une part, l'enseignement d'autre part. Tous deux ont connu depuis la révolution anthroponomique de la fin du $\mathrm{XIX}^{\mathrm{e}}$ siècle (Bertaux 1994) un essor absolument considérable, et des transformations profondes qui n'ont jamais cessé. Malgré son très long confinement dans le cadre des foyers, où elle était régie par des rapports sociaux de sexe fonctionnant comme rapports de production anthroponomique, cette seconde production a fini par s'en émanciper et a connu dès lors ce qu'avait connu un siècle plus tôt - à la fin du XVIII ${ }^{\mathrm{e}}$ siècle donc - la production des choses: une révolution ininterrompue de ses forces productives.

À la différence que ce n'est ni dans la forme du marché simple, ni dans celle de la forme capitalistique (rap- port capital / travail salarié) qu'elle a trouvé à s'épanouir; mais, de façon totalement contre-intuitive pour la pensée unique, dans la forme publique que nous connaissons bien en France 5 .

Dans les pays les plus avancés ces deux derniers secteurs d'activité rémunérée constituent à eux seuls environ $20 \%$ du Produit Intérieur Brut $^{6}$. Ils emploient autour de $15 \%$ de la population active, mais plus de $35 \%$ de sa partie la plus qualifiée. Ces chiffres suffisent à indiquer qu'il ne s'agit aucunement de secteurs marginaux, improductifs, archaïques... Bien au contraire, alors que dans ces pays la production industrielle continue à perdre des emplois (sous les effets conjoints de l'automatisation surtout - et de la délocalisation des usines) ces deux secteurs d'activité ainsi que d'autres secteurs de la production anthroponomique tels le care aux personnes âgées - continuent à se développer rapidement. Ce sont eux qui tirent la croissance des services; la 'bonne' croissance, la croissance utile à l'ensemble de la population, par contraste avec celle des services financiers ${ }^{7}$.

\section{Des services productifs}

Les activités de production anthroponomique se présentent toutes comme des services aux personnes. Or on rencontre encore beaucoup d'économistes qui ne considèrent pas les services comme faisant partie de la production matérielle. Pour mémoire, rappelons qu'au milieu du XVIII ${ }^{\mathrm{e}}$ siècle Quesnay et les physiocrates pensaient que seule l'agriculture était productrice de richesses...

Les services anthroponomiques opèrent sur le corps (au sens de Michel Foucault: le corps physicopsychique, le soma-psyché, ce que nous appellerons ici le corps total); et ils contribuent ainsi, très directement, à la production matérielle des membres d'une société donnée. Ils produisent et re-produisent quotidiennement leur énergie vive, en les nourrissant par exemple; ils les maintiennent ou les remettent en bonne santé; ils accroissent les formes quali- 
fiées des énergies vives, les savoir-faire et savoirs incorporés, dont les corps totaux sont porteurs; et par un travail sur le sens, ils renouvellent leur 'niveau de moral', leur énergie morale, pour leur éviter de sombrer dans l'apathie et la dépression. En résumé ils produisent la vie même, et les chances d'une vie bonne.

Certes l'analogie entre production des choses et 'production' des gens eux-mêmes choque au premier abord, et même doublement: du point de vue cognitif comme du point de vue normatif.

$\mathrm{Du}$ point de vue cognitif, la première réaction de sens commun sera de réduire l'idée de 'production des gens' à une métaphore. Toute activité de production, dira-t-on, qu'elle soit agricole, artisanale, industrielle, produit des résultats tangibles; des objets qui peuvent être vus, photographiés, comptés, pesés, mesurés, vendus (ou échangés) et consommés. Par contre les activités des services anthroponomiques semblent ne rien produire de tangible: leurs effets supposés sur les 'corps' ne se voient pas; ils ne peuvent être ni photographiés, ni comptés, ni pesés, ni mesurés, et encore moins vendus ou échangés. Ils ne sont pas séparables des corps totaux dans lesquels ils se fondent. Ils n'auraient aucune existence matérielle indépendante, et il ne pourrait donc s'agir de production au vrai sens du terme.

Pourtant que serions-nous, chacun de nous, sans l'accumulation continue et la sédimentation, dans notre 'corps total', des effets des actions anthroponomiques (y compris les actions de care) que de nombreuses personnes ont faites pour nous, sur nous, avec nous? À commencer par notre mère bien évidemment, dont le corps a objectivement produit le nôtre mais qui en tant que sujet nous a subjectivement accepté, attendu, porté longuement, et finalement mis au monde. Puis elle nous aura 'élevé' pendant une longue période. Nous ne lui devons pas seulement la vie une seule fois, mais de multiples fois; et, en bonne part, ce que nous sommes devenus.

Or elle n'aura été que la première de toute une série d'agents anthroponomiques qui se sont occupés de nous tout au long de nos années d'enfance, puis d'adolescence, jusqu'à que nous devenions plus ou moins capables de nous assumer nous-mêmes. Le père, bien sûr, et d'autres membres du groupe familial auront beaucoup compté. Mais bien d'autres personnes aussi se sont occupées de nous, nous ont maintenu en vie et nous ont fait grandir: puéricultrices, éducatrices et éducateurs de maternelle, maîtresses d'école et instituteurs, enseignants du secondaire, y compris les professeurs d'éducation physique et sportive, médecins et infirmières, coiffeuses, psychothérapeutes, et tant d'autres, sans oublier notre propre action en tant que producteurs de nous-mêmes...

Tous ces acteurs ont contribué intentionnellement ou non - à donner forme à notre corps-total, à le maintenir en vie et en bonne santé, à l'emplir de contenus les plus divers (que nous avons plus ou moins bien appris et digérés); à faire de nous, répétons-le, ce que nous sommes devenus aujourd'hui et ce que nous en ferons. Sans leurs actions nous serions peut-être déjà morts plus d'une fois; et si par miracle nous avions réussi à survivre, c'eut été non seulement en tant qu'analphabètes mais sans même avoir acquis la capacité d'articuler un seul mot, voire de se tenir debout. Le montre assez le célèbre exemple de l'enfant sauvage de l'Aveyron, capturé en 1800 à l'âge d'environ 12 ans; à l'origine sans doute un bébé abandonné par sa mère dans la forêt, recueilli et allaité par une louve, courant à quatre pattes, se nourrissant de racines, et que le brave médecin Itard ne parvint jamais à 'civiliser'...

Le care n'est donc pas le tout de la production des gens eux-mêmes, loin de là; il n'en est que la partie aujourd'hui émergée, rendue enfin visible par Carol Gilligan. Au-delà du care existe un immense système en mouvement, organisé, diversifié, complexe, fonctionnant à plein régime: c'est ce système des activités de production anthroponomique, ce système anthroponomique (comme on dit "système économique") dont les logiques de fonctionnement, les tensions internes et externes et les dynamiques de transformation restent entièrement à analyser et à comprendre.

\section{Anthroponomie et économie matérielle}

La production 'des gens eux-mêmes', la production anthroponomique, et la production des choses (l'économie matérielle) sont très fortement imbriquées l'une dans l'autre. Car l'une ne va pas sans l'autre, et chacune de ces deux productions, vraies jumelles inversées en quelque sorte, se nourrit de et consomme ce que l'autre produit, non seulement quotidiennement mais aussi à moyen et long termes; une consommation grâce à laquelle elle peut continuer à produire ce que l'autre va consommer (Bertaux 1977, ch. 2).

Ainsi va le monde. Mais l'énigme fascinante est que, tout en étant aussi étroitement imbriquées l'une avec l'autre, l'une dans l'autre, ces deux grandes formes de production - la production des choses et la production des gens - ne suivent absolument pas la même logique. L'une a désormais pour but à peu près exclusif l'argent, un concept évidemment bien plus complexe qu'il n'y paraît à partir du moment où l'argent prend la forme de capital, de crédit et de la finance; l'autre a pour but premier la vie, un concept plus complexe encore. Et ces deux étoiles polaires, ces deux 'déesses' sont en tension permanente l'un avec l'autre, bien qu'elles se nourrissent en permanence l'une de l'autre.

Si l'on rencontre encore à la surface de la planète des microsociétés sans écriture (et sans rapports de classe) où l'on ne 'travaille' à produire des choses que dans la mesure où elles sont nécessaires à la vie, mais où la priorité est clairement la vie et non le travail, dans notre civilisation au contraire l'équilibre a basculé sur l'autre bord: l'économie a colonisé l'anthroponomie, et l'on en vient, globalement parlant, à vivre pour travailler. Mais il arrive encore que l'on se rappelle qu'elle ne devait et ne devrait être qu'un moyen, et non une fin en soi; et cette tensionlà est porteuse de grandes transformations présentes et futures. 
Considérant dans la suite que le care a déjà été longuement présenté et discuté ailleurs nous nous focaliserons ici sur le concept de production anthroponomique qui l'englobe. Comme un baleineau en pleine croissance cette 'production des gens eux-mêmes' ne cesse de grandir et de se diversifier: il devient urgent de la penser en tant que telle et de chercher à saisir les logiques, les tensions internes et les dynamiques de transformation qui lui sont propres.

Nous centrerons ici notre attention sur ses divers modes d'organisation: la diversité des formes (les rapports sociaux de classe, de genre...) qui la structurent et l'organisent en tant que ses rapports de production.

\section{Reproduction ou production anthroponomique?}

Peut-on parler de 'production' stricto sensu quand il s'agit de produire non pas des choses mais des personnes? Le terme de production signifie l'application d'une force qui travaille sur un matériau - brut ou intermédiaire - dans l'intention de le transformer en produit utile (intermédiaire ou fini) à utiliser (consommer directement) ou à échanger (vendre). Mais si ce matériau est un être humain, une personne, cette définition tientelle encore, et au prix de quels changements conceptuels?

Or le second type de production travaille bien sur un 'matériau': c'est le corps. Un corps qui ne se réduit pas à sa composante physique mais englobe aussi (et d'abord) sa composante psychico-mentale, ainsi que les relations entre soma et psyché. C'est le corps physico-psychique, la machine corporelle-spirituelle vivante, consciente, volontaire et souffrante, la "machine désirante" décrite par Deleuze et Guattari (1972): le corps total.

Comme dans la production matérielle des choses, qui - à part l'agriculture, et encore - ne produit rien ex nihilo mais travaille toujours sur de la matière première, il ne s'agit pas de produire ce corps total à partir de rien. Une femme, sa future mère, s'en est chargée, comme on l'a rappelé: pendant que des processus naturels au sein de son propre corps fabriquaient un nouvel être, elle en a accompagné la croissance jusqu'à ce qu'au prix d'une intense souffrance elle 'mette au monde' ce nouveau vecteur de vie humaine, dont l'orbe de vie potentielle s'est élevée d'emblée bien au-delà de son propre horizon temporel.

Mais ce n'est pas tout: ce dont il s'agit ensuite, c'est d'en faire un être humain socialisé, apte à vivre en société, ce qui va demander un travail considérable de la part de nombreux acteurs. Comme l'écrit Pierre Legendre: «Il ne suffit pas de produire de la chair humaine, encore faut-il l'instituer, c'est-à-dire nouer le biologique, le social et l'inconscient par des moyens juridiques qui fassent loi généalogique pour le sujet » (Legendre 1985).

La machine vivante que nous habitons est vorace: elle a besoin de produire quotidiennement l'énergie nécessaire à ses activités non seulement physiques mais aussi psychiques et mentales. Cela ne se fait pas tout seul; cela demande, quotidiennement, un travail de (re)production.

Si cependant nous tenons à parler de production plutôt que de 'reproduction', comme c'est l'usage, c'est parce que le changement y est la règle; et la reproduction à l'identique, l'exception. Le cas général c'est la découverte quotidienne de nouveautés, et pas seulement chez l'enfant; ce sont les multiples apprentissages qui doivent être faits en permanence pour que le corps-total s'adapte à un environnement en mouvement et acquière les capacités, les habiletés, les skills nécessaires pour pouvoir faire ce que veut la personne qui l'habite (ainsi, pour l'enfant, se tenir debout, marcher sans tomber, parler...) et/ou ce que son entourage lui demande de faire. Un mode de vie quotidienne qui se limiterait à de la reproduction à l'identique, sans stimulations sensorielles, sans nouveauté aucune, sans contacts, sans encouragements moraux, serait profondément démoralisant et déstabilisant. S'inspirant des travaux de Damasio, Carol Gilligan (2009) a rappelé dans un entretien récent combien le rationnel (le 'mental') est intime- ment lié à l'affectif (le 'psychique') au sein du corps total.

La matière sur laquelle travaille la production anthroponomique est certes d'une grande complexité... Mais notre approche, sociologique, est centrée sur les rapports socio-structurels ${ }^{8}$ qui sous-tendent et organisent cette seconde 'production'; et cette question est fort heureusement d'un ordre de complexité bien inférieur.

\section{'Production' ou prod/action?}

Toute action de 'production' anthroponomique implique (au moins) deux personnes, dont l'une est en position de 'sujet' et l'autre en position - temporairement du moins - d'objet'; de 'matériau' à transformer par cette action. C'est donc a priori une situation typique d'un rapport de pouvoir entre deux personnes. Le bébé, l'enfant, la personne handicapée, la personne très âgée ont des besoins matériels primaires - la faim par exemple - qu'ils ne sont pas en mesure de satisfaire par eux-mêmes, sans aide. Ils sont ainsi dépendants de l'acteur de care ${ }^{9}$.

Or l'expérience montre que la personne en situation d'objet ne se transforme qu'à la condition qu'il / elle participe activement à sa propre transformation, donc en tant que sujet. Pratiquement aucune des activités de production anthroponomique que nous avons examinées n'atteint son but sans la participation active de son 'objet'. Peut-on faire manger un bébé qui n'a pas faim et refuse d'ouvrir la bouche? Peut-on enseigner quelque chose à un enfant, un élève ou un étudiant qui ne veut pas apprendre? Peut-on soigner un(e) malade qui n'a pas envie de guérir, voire de vivre? ${ }^{10}$

C'est pourquoi il conviendrait sans doute de parler de premier et de second sujet, plutôt que de sujet et d'objet. Et au lieu de parler de 'production', parler de prod/action (mais comme aurait pu dire Barthes, la parole est spontanément xénophobe: elle rejette a priori les mots étrangers...)

Quant aux 'transformations' du second sujet, il faut distinguer les transformations visées (voulues, inten- 
tionnelles) par l'action de prod / action anthroponomique, et ses transformations effectives qui sont souvent fort différentes...

De façon générale la question de la prod/action anthroponomique est: qui fait quoi, à qui, où, quand, comment - dans quelles conditions, avec quels moyens -, dans quelles intentions (apparentes, déclarées vs. réelles mais non-dites). Avec quels résultats, quels effets réels (éventuellement nonvoulus) à court, moyen et long terme: apprentissages, expériences (bonnes ou mauvaises). Et avec quel degré de consentement du 'second sujet'... voire du premier sujet quand il / elle se trouve contraint, par la nécessité économique ou par une relation de pouvoir politique, de rendre des services anthroponomiques contre son gré.

Il est donc tout à fait évident que dans le care, et plus généralement dans toute action de prod/action anthroponomique, la question éthique se pose toujours à quelque degré. Mais inversement on ne saurait réduire ce type d'activité à cette question: elle n'en constitue qu'une des dimensions. Il suffit de concevoir les situations de care sociologiquement, c'est-à-dire à partir des rapports sociostructurels qui les définissent (et qui sous-tendent les interactions interpersonnelles qui s'y développent) pour constater qu'en termes d'éthique elles varient considérablement.

Soit un exemple concret, celui d'un bébé qui a faim. À un extrême du continuum de solutions à ce 'problème', c'est sa mère qui va se charger de le nourrir. Elle le fait - dans la majeure partie des cas - avec une infinie tendresse et un bonheur sans égal. Sa motivation est sans limites; et la logique psychologique, affective, qui régit sa relation à son enfant est celle du don, sans même l'ombre d'une attente d'un hypothétique contre-don (Mauss 1923-24). On est ici dans l'éthique du care telle que la conçoit Carol Gilligan.

Pourtant la logique socio-structurelle qui sous-tend cette relation se situe sur un tout autre registre. Ce n'est nullement une logique psychologique; ce n'est pas non plus, malgré les apparences, une logique éthique. C'est une logique sociale: c'est le registre d'un ordre social institué, en l'occurrence celui de la division sexuelle du travail anthroponomique, qui s'incarne normativement dans l'encadrement juridique de la distribution des responsabilités. L'ordre social assigne chaque mère au care de son enfant; il lui attribue ainsi la pleine responsabilité (non seulement morale mais juridique) de son bien-être, de son état, qu'elle le veuille ou pas. Une chatte peut abandonner l'un de ses petits, voire toute la portée; une femme ne le peut pas, car elle s'exposerait à une sanction extraordinairement lourde. Elle peut seulement déléguer la tâche à un(e) autre; mais ne peut se défaire de sa responsabilité ultime.

Considérons maintenant le cas d'une femme qui tient à avoir un enfant, mais qui considère qu'elle a mieux faire que de s'en occuper, et qui a les moyens de le confier à une autre femme qui sera nourrie-logée (et surveillée) sous son toit. Récurrente à travers les siècles et les modèles culturels, cette situation aboutit souvent à ce que ce soit à une jeune fille d'origine rurale - voire de nos jours une immigrée en situation irrégulière - que sera confié le bébé. De nombreux auteur(e)s ont étudié ce type de situation structurée par une combinaison de rapports de genre et de classe; auxquels s'ajoutent aux États-Unis (et ailleurs) des rapports de 'race' (Nakano Glenn 2010). Elle s'accompagne fréquemment d'une exploitation parfois éhontée du travail de la jeune fille. Mais ce n'est pas là une question d'éthique: c'est une question de droits sociaux et de droits syndicaux consécutifs à des rapports de force collectifs entre employeurs et employée(s) dans une société donnée. Sur ce point la Suède, et la France avec le Chèque emploi service, sont en avance sur bien d'autres pays.

Mentionnons enfin la possibilité objective de maltraitance dans les maisons de retraite pour personnes très âgées: ce cas se situerait à l'autre extrême du continuum de l'éthique du care.

On voit sur ces exemples que ce n'est ni le contenu intrinsèque de la tâche de care ni la question éthique qui pourraient permettre de connaître à l'avance la configuration des situations de care: c'est la forme spécifique du cadre de rapports sociostructurels instituant une telle situation. C'est cette forme qui définit et distribue les tâches à accomplir, les responsabilités, les récompenses et gratifications (rewards) et les sanctions entre les divers agents / acteurs impliqués, différemment situés les uns par rapport aux autres dans la répartition des tâches. Aussi leurs relations interpersonnelles et leurs interactions mutuelles, tout en variant selon la 'différentialité' (Bertaux 2010) des personnes (leur histoire, leur caractère...) ne peuvent qu'être fortement dépendantes des places respectives dans lesquelles ils se trouvent assignés, de la répartition des tâches et des responsabilités entre ces places, des rapports entre ces places occupées par les différents acteurs: rapports de force sur le marché du travail, rapports de commandement et d'obéissance, d'autorité, de contrôle, d'alliance...

Ce que nous voulons dire, c'est qu'à partir du moment où l'on considère le care comme une activité productive on ne peut plus se contenter de l'analyser en termes d'éthique. Et cela est encore plus vrai du très vaste ensemble d'activités de production anthroponomique dont le care ne constitue aujourd'hui que la partie émergée, la partie visible. Que chacune de ces activités, parce qu'elle travaille directement sur le corps-total d'une personne, présente une composante éthique, cela va de soi: il existe une éthique médicale, une éthique de l'encadrement de jeunes enfants, une éthique de l'enseignement primaire, une éthique du soin aux handicapés... Mais ce qui nous intéresse ici, ce sont les questions d'organisation des activités de production anthroponomique, et les formes alternatives que prend cette organisation.

\section{Analyser les différentes formes de la production anthroponomique}

L’histoire de la pensée économique montre la voie: c'est en analysant patiemment la variété des formes que prenait la production (et la distribu- 
tion) de richesses qu'elle a pu aller de l'avant.

Pour reprendre l'exemple de la prise en charge d'un bébé, deux formes différentes d'organisation du travail de care ont été décrites plus haut. La première est sa prise en charge par sa mère dans un cadre de rapports familiaux; c'est la forme familiale. Si la jeune mère confie son bébé à sa propre mère ou au père de l'enfant on reste encore dans le même cadre 'familial', donc aussi dans la même sphère normative et symbolique, dans la même économie morale construite sur la base de relations de parenté, d'intimité, d'attachements, de circulation de dons créant des sentiments (variables) de dette et d'éventuels contre-dons; du sentiment d'appartenance à un même petit groupe d'entraide et de solidarité. L'enfant est l'enfant du groupe familial.

La deuxième forme d'organisation est fort différente, puisque c'est une personne extérieure au groupe familial à qui est confié le bébé pour qu'elle fasse pour lui le travail de care impérativement requis, contre rémunération en nature ('nourrie-logée') et/ou en argent. Elle effectue ainsi une activité de service en échange d'une rémunération, mais sans intermédiaire extérieur, donc sous forme marchande simple. Elle peut être remplacée par une voisine, ou quand l'enfant grandit par une jeune fille au pair; mais la forme reste grosso modo la même.

Mais d'autres formes d'organisation du travail de care existent: des crèches entièrement privées, non subventionnées, qui fonctionneraient comme des entreprises tournées vers le profit, relèveraient d'une forme capitalistique (en anglais for profit). La littérature anglo-saxonne sur le care tend souvent à confondre cette forme avec la forme marchande simple sous la même appellation de market, ce qui du point de vue analytique nous semble erroné.

Avec des crèches fortement subventionnées on entre dans une autre logique encore, que les mêmes auteures anglo-saxonnes désignent par State (État): nous préférons l'appeler forme publique. Cette forme est très répandue dans les pays scan- dinaves-nordiques, et à un moindre degré en France même. Elle y joue un rôle essentiel: en effet elle permet aux mères de jeunes enfants de garder leur emploi; de plus elle crée de nouveaux emplois de salarié(e)s; et ce n'est pas un hasard si la natalité est à l'heure actuelle significativement plus élevée dans les pays à crèches publiques et écoles maternelles que, par exemple, en Allemagne ou dans les pays du Sud de l'Europe. Le financement de la forme publique est assuré par l'impôt, quel que soit le niveau auquel il y est recueilli (municipalité, département, région, nation...).

Dans une longue étude mentionnée plus haut l'économiste du développement Shahra Razavi a proposé en 2007 une typologie de quatre formes d'organisation du care, qu'elle a désignées par Familles / ménages, Marchés, État (fédéral/local), et 'Not-for-profit' (les associations bénévoles et/ou caritatives) ${ }^{11}$. Inspirée par cette typologie, la sociologue japonaise Emiko Ochiai a effectué avec l'aide de chercheurs locaux une vaste enquête dans six pays d'Asie sur les formes de prise en charge des enfants en bas âge (les modes de garde, le childcare). Elle a ainsi pu montrer que, selon les pays et les régions, les quatre sommets du 'losange du care' sont très inégalement utilisés (Ochiai 2009). Dans les zones rurales très densément peuplées la prise en charge des jeunes enfants est encore, conformément à la tradition, l'affaire des mères et d'autres femmes du groupe familial. Mais dans les villes et les grandes métropoles asiatiques une nouvelle tendance se dégage: un nombre croissant de jeunes femmes éduquées rejettent le modèle de la femme / mère au foyer et désirent à la fois avoir un ou deux enfants et garder leur emploi. Elles font alors appel aux services de jeunes filles venues de la campagne, ou à une offre for-profit de crèches privées qui se développe très vite. Ochiai, qui connait bien la fonction jouée par les crèches et maternelles en Suède et en France, regrette qu'en Asie cette forme publique soit encore si peu connue et pratiquée. La Chine communiste était le seul grand pays où une forme apparentée, la forme étatique, avait été diffusée massivement mais de façon autoritaire et très contraignante, pour 'libérer' la main-d'œuvre féminine et la mobiliser dans des grands travaux collectifs. Elle y est désormais en grand déclin ${ }^{12}$.

Ainsi, comme pour la production des choses - on se souviendra peutêtre du fameux débat qu'avait lancé Louis Althusser sur les 'modes de production' selon Marx - il y a plusieurs grands types de 'modes de production de la vie'. Mais à la différence des premiers qui - schématiquement - se succèdent dans la très longue durée de l'Histoire en fonction du développement des forces de production, les seconds peuvent coexister au sein d'une même société.

Pendant très longtemps cette production de la vie s'est faite dans le cadre familial, et donc à l'intérieur même de chacun des groupes sociaux - caste, 'ordre', corporation, 'classe' - composant la 'société' locale; chaque groupe préparant ses enfants (en fonction de leur sexe) à succéder à leurs parents. Dans les familles de 'producteurs directs' les enfants étaient élevés sur le tas, mêlés aux activités des adultes; dans celles des 'maîtres de conditions de production', de nombreux serviteurs faisaient office de producteurs anthroponomiques directs; et dans les couches intermédiaires - ces classes 'moyennes' que Sartre (1971, tome I) qualifie joliment de classes-moyens car leur fonction était de relayer le pouvoir des maîtres - on s'efforçait d'imiter les supérieurs. Jusqu'à la fin du XIX ${ }^{\mathrm{e}}$ siècle c'est donc la forme familiale, déclinée selon les milieux sociaux, qui prédomine presque partout.

Tout a changé avec la révolution anthroponomique qui a institué la scolarisation, les premiers hôpitaux modernes, les premières institutions de prise en charge des personnes dépendantes. Les formes de la production anthroponomique se multiplient: Sharah Razavi, focalisant son attention sur le childcare, en distingue quatre principales. En distinguant, au sein de sa catégorie market, la forme marchande simple et la forme for-profit, et au sein de sa catégorie State la forme étatique 'pure' et la forme publique nous arrivons à six. Elles coexistent toutes, mais y en a-t-il une qui se 
dégage comme mieux adaptée que les autres à la 'production de la vie', et donc plus porteuse d'avenir?

\section{Forme marché ou forme publique?}

S'il s'agissait de la production matérielle des choses, le sens commun contemporain répondrait aussitôt: la question a été tranchée par l'Histoire en faveur de la forme-marché. Ou pour être plus précis, la forme industrielle for-profit, celle de la grande organisation à capitaux privés (aujourd'hui de plus en plus soumise à la logique financière). Les expériences 'communistes' que l'Histoire a conduites en URSS puis en Chine et à Cuba ont certes permis le décollage économique de pays sous-développés - à un coût humain prohibitif - mais se sont finalement soldées par des échecs économiques (et politiques). L'Histoire donnerait donc raison à Friedrich von Hayek: il était certain d'avoir démontré une fois pour toutes que la concurrence libre et non faussée, et 'le marché' qui en résulte partout, constituent de facto une gigantesque et extraordinairement efficace 'machine' de traitement en temps réel d'une masse considérable d'informations, et donc de formation des justes prix de toutes les marchandises. Aucun système centralisé et planificateur, aussi sophistiqué et numérisé soit-il, ne saurait jamais faire aussi bien en rapidité et en précision.

Autrement dit, au rebours des grands récits sur l'égalité, la cohésion sociale et autres grands idéaux, l'Histoire aurait montré que la production des choses se fait mieux dans le cadre de la forme-marché13.

Mais en est-il de même de la production de la vie? Nous avons la conviction que pour elle c'est précisément le contraire: la forme-marché y est bien moins adaptée qu'une autre forme, la forme publique ${ }^{14}$.

Plusieurs historiens et sociologues se sont penchés, à propos de tel ou tel pays, sur l'histoire de son système d'enseignement, de son système de santé, ou de ses institutions de protection sociale: soit les trois grands piliers institués de la production anthropo- nomique. Le sociologue hollandais Abram de Swaan a repris ces séries d'études pour cinq pays: Grande-Bretagne, France, Allemagne, Pays-Bas, et de façon moins détaillée, États-Unis. Dans son ouvrage In Care of the State (de Swaan 1988; trad. fr. Sous l'aile protectrice de l'État) il reprend en détail ces histoires parallèles d'institutions qui, apparues au gré de contingences locales, ont presque toutes fini - sauf aux États-Unis - par converger vers une même forme: celle, familière aux Français, que nous appelons ici la forme publique.

Les premiers embryons de ces futures institutions sont souvent apparus à l'initiative de groupes locaux, donc sous la forme associative: telle Église majoritaire organisant la charité dans le périmètre de sa paroisse; tel syndicat ouvrier créant, sur la base de cotisations volontaires, des caisses de solidarité mutuelle contre les risques d'accident, de chômage, de perte de la capacité de travailler en raison de l'âge; telle Église minoritaire - protestante en général - cherchant à développer l'apprentissage de la lecture pour diffuser la connaissance de la Bible... Puis ces formes se sont étendues et ont considérablement évolué avec le temps, en suivant des cheminements singuliers, parfois tortueux, non exempts de retours en arrière; en changeant d'échelle elles ont cherché des formes plus 'rationnelles'. Or dans les quatre pays européens étudiés leurs trajectoires historiques, bien que ballotées par les jeux des intérêts et les circonstances, les ont presque toutes amenées à prendre la forme publique; ce qui, dans des contextes d'économie de marché pourtant très hostiles à cette forme, est d'autant plus remarquable.

Ainsi en France, des initiatives diverses cherchant à diffuser l'enseignement de la lecture ont-elles fini par rencontrer, dans les années 1870 , la visée d'un État décidé à unifier linguistiquement (et à terme, culturellement et politiquement) un peuple 'français' composé d'une grande majorité de ruraux ne parlant que le dialecte de leur région et ne pouvant se comprendre entre eux (Weber 1976). D'où le projet de couvrir le territoire d'écoles primaires laïques (au grand dam de l'Église catholique), obligatoires pour tous les enfants, et gratuites d'accès car financées par l'impôt: ce qui plaçait toutes les familles de la République sur un plan d'égalité formelle, une caractéristique typique de la forme publique.

Autre exemple: en Allemagne dans les années 1880 le succès rencontré auprès des ouvriers qualifiés par les caisses de solidarité mutuelle mises sur pied par leurs syndicats, leur diffusion rapide et surtout le surcroît de légitimité ainsi gagné par les organisations ouvrières et socialistes inquiétèrent le Chancelier conservateur Bismarck. Suffisamment pour qu'il imagine et développe un système concurrent, étatique celui-là. Un système d'assurances mutuelles à l'échelle de tout un pays, basé sur le recueil de cotisations obligatoires donnant droit à des pensions en cas d'accident, puis aussi de maladie, puis d'invalidité, et même finalement à une (toute petite) retraite. Ce n'était pas par philanthropie mais - disait Bismarck - pour "forger une mentalité conservatrice dans la masse des plus démunis". Cette opération de récupération, menée en parallèle avec une répression féroce des syndicats et associations socialistes, visait à les priver du surcroît de légitimité dû à leur initiative, et surtout du dynamisme consécutif à la participation de nombreux volontaires à la gestion des mutuelles. Le coup fut un succès: car la bureaucratie, même celle d'un État impérial tenu par une haute aristocratie très anti-ouvrière, présentait en matière de gestion de forts avantages sur une mutuelle ouvrière: parce que tous les bénéficiaires potentiels étaient obligés de cotiser (solution radicale au problème du free rider qui affaiblit les associations bénévoles); parce que la bureaucratie d'État disposait de spécialistes des calculs actuariels pouvant évaluer au plus juste les coûts collectifs des risques et donc le montant des cotisations individuelles; et parce que l'État serait encore là dans dix ans, vingt ans, trente ans... Les principes rationnels de ce système ont été repris en France par le Conseil national de la Résistance et ont donné naissance en 1945 à notre Sécurité Sociale; d'autres 
pays d'Europe continentale s'en sont aussi inspirés.

Ainsi de Swaan constate que dans chacun des quatre grands types d'activité qu'il a choisis d'étudier: enseignement, santé, protection sociale, réseaux urbains d'égouts - qui relèvent tous directement, sauf le dernier, de la production anthroponomique - les premières initiatives sont venues d'associations locales. Puis les premières formes d'organisation ont connu de nombreuses métamorphoses, au gré de luttes d'intérêt et de mobilisations aux issues incertaines. Mais au terme de ces tâtonnements et pérégrinations elles semblent toutes converger vers une même forme, la forme publique. La confirmation de l'existence d'une logique immanente à l'objet lui-même est d'autant plus forte que De Swaan n'est pas un théoricien attaché à étayer/démontrer sa thèse par une lecture sélective de l'Histoire, mais un disciple direct de Norbert Elias, historien et sociologue inspiré par Max Weber.

À l'hypothèse d'une affinité structurelle entre production anthroponomique et forme publique on peut cependant opposer une exception de taille: celle des États-Unis. À première vue l'hypothèse ne s'y vérifie pas. Ce sont des institutions privées fortement capitalisées qui organisent l'essentiel du marché des soins médicaux. Toutes les universités, en concurrence féroce entre elles, sont d'accès payant. Le système de retraite, très diversifié, repose pour l'essentiel sur le principe de l'épargne individuelle (capitalisation) confiée à tel ou tel fonds de pension qui la place en Bourse. Le pays est profondément libéral et individualiste; son modèle culturel est tout entier construit autour de l'idéal de liberté. Toute forme d'organisation collective - ou pire, étatique - est immédiatement assimilée dans le subconscient sociétal à un 'collectivisme' évoquant le communisme totalitaire. Autant dire le diable.

On peut donc considérer la société états-unienne comme une expérimentation en vraie grandeur, celle des qualités et défauts de la forme for-profit pour ce qui est de l'organisation des services de production de la vie. Est-ce qu'elle fonctionne bien?

Commençons par le système de santé. Au cours des dernières années les débats sur les projets d'Hilary Clinton, puis de Barak Obama pour le réformer ont apporté beaucoup d'informations. Ce système est pour l'essentiel aux mains de très grandes compagnies privées d'assurance-maladie. Elles s'entendent entre elles sur les tarifs, et cherchent à n'assurer - c'est le paradoxe - que des individus en bonne santé, n'hésitant pas par exemple à refuser de renouveler l'assurance d'une personne qui a déjà été victime d'un infarctus. Plus de 40 millions d'Américains ( $15 \%$ de la population) n'ont pas d'assurance médicale, presque autant sont pauvrement assurés; et ils seraient beaucoup plus nombreux encore si le Président Johnson n'avait réussi à faire voter en 1965 la création de deux systèmes de prise en charge publique d'une partie des frais médicaux, ceux des personnes de plus de 65 ans (Medicare, $16 \%$ de la population) et ceux des ménages aux revenus les plus bas (Medicaid, 14\%). Piètres performances finalement, d'autant que l'ensemble des dépenses de santé équivaut à $18 \%$ du PIB aux États-Unis, contre $11 \%$ en moyenne en Europe de l'Ouest pour une 'couverture' quasiuniverselle et un meilleur état général de santé. La comparaison tourne donc au net avantage de la forme publique, celle qui organise en Europe - avec quelques variantes - l'essentiel du système de soins médicaux des pays les plus développés.

La forme marché fait-elle mieux, au vu de l'expérience américaine, en termes d'enseignement - donc de production des chances de vie pour les enfants d'un pays donné? Notons d'abord qu'aux Etats-Unis la scolarisation est massive, mais qu'elle y est réalisée dans des établissements primaires et secondaires d'accès gratuit, car financés par les impôts; donc dans une variante, certes beaucoup plus décentralisée qu'en France, de la forme publique. Ce sont les études supérieures qui sont payantes, et l'offre en est extraordinairement diversifiée, en prix comme en qualité. Mais - comme en Europe - le 'prix de revient', le coût minimal, pour une Université, d'une année d'études supérieures est de $10000 \$(8000 €)$ par étudiant; un coût que seules peuvent payer les familles riches ou celles, relativement aisées, pouvant se porter garantes d'un emprunt étudiant. Les universités le vendent évidemment plus cher, parfois beaucoup plus cher. Chacune cherche à attirer à elle les meilleurs enseignants en leur offrant de bons salaires (et donc en augmentant les frais d'inscription), mais chacune veut aussi, pour consolider sa réputation dans la durée, recruter les meilleurs étudiants des États-Unis et du monde entier, dont beaucoup ne viendront que si on leur propose une bourse... Cette tension entre deux objectifs contradictoires débouche sur une grande diversité de réponses institutionnelles, des très nombreux mais modestes colleges (premier cycle universitaire) aux universités les plus chères et les plus prestigieuses, en passant par les universités d'État (Californie, Texas...) et des instituts spécialisés. Mais ce qu'il faut retenir c'est que pratiquement tous ces établissements fonctionnent en partie grâce à des fonds publics: les cinquante universités d'État sont évidemment subventionnées par leur État; mais les universités totalement privées, y compris les plus connues, ne bouclent leur budget que grâce à de très généreuses donations de compagnies privées ou d'anciens élèves devenus millionnaires, qui bénéficient ainsi de fortes déductions fiscales consenties par l'État fédéral ${ }^{15}$.

Quant à la prise en charge des personnes dépendantes par la collectivité, et notamment les personnes âgées et / ou malades, elle n'est bien entendu pas le point fort, et c'est un euphémisme, du système institutionnel états-unien. De très nombreuses associations d'une société civile encore très active tentent d'y remédier - comme d'ailleurs pour les soins médicaux aux non-assurés - mais elles ne peuvent empêcher des millions de gens de passer avec l'âge d'une pauvreté digne à des situations de misère. Le mariage de la vie avec le commerce ne donne pas que de beaux enfants.

Ce rapide tour d'horizon conduit à un constat minimal: comme pour 
la production des choses (l'économie matérielle), la production des gens eux-mêmes, la production de la vie et des chances de vie peut être organisée de diverses façons, sous diverses formes. Mais si, pour la production des choses, les formes marchandes ont fini par s'imposer comme les plus efficaces $^{16}$ - sinon les plus justes, tant s'en faut-il ne semble pas en être - de même pour la seconde production, dont fait partie le care: là c'est manifestement la forme publique qui semble donner les meilleurs résultats ${ }^{17}$.

Pourquoi en est-ilainsi? En vertu de quelle(s) logique(s) sociologique(s) immanente(s)? Telle est la question que nous examinerons brièvement pour terminer.

\section{Discussion}

Tant les conclusions d'Abram de Swaan que l'examen d'alternatives semblent confirmer l'hypothèse de la tendance des grandes activités anthroponomiques à prendre la forme publique plutôt qu'une forme marchande for-profit.

À quoi pourrait ressembler cette logique immanente? On peut faire l'hypothèse qu'au fur et à mesure qu'un groupe humain se structure en 'société' stabilisée, qu'il s'autoorganise via un ensemble de rapports sociostructurels coutumiers ou institués qui sont aussi et d'abord des rapports de pouvoir, ses dominants/dirigeants imposent à chaque famille de fournir des ressources pour 'le bien commun'. Ce centre dirigeant (et dominant) peut selon les époques s'incarner dans la chefferie, le château, le roi, l'empereur, l'Église, la municipalité, l'État... : quelle qu'elle soit, c'est cette instance centrale qui est tenue pour responsable $d u$ bien commun. Si la vie d'un des membres de cette société locale se trouve menacée en raison d'un accident, de la pauvreté, de la maladie et si ses proches n'ont pas les moyens de l'aider ils se tourneront spontanément vers le 'centre', qui dispose d'importantes ressources. Certes la vie individuelle n'a pas toujours eu l'extraordinaire charge symbolique qu'elle a pris de nos jours, du moins en Occident; mais la vie humaine a de tout temps été plus ou moins fortement associée au sacré.

Bien entendu un tel processus présuppose qu'une sorte d'économie morale cimente le groupe en question; et notamment une économie du sentiment de solidarité à l'égard des autres membres du groupe en tant que tels, qu'on les connaisse personnellement ou pas. Un risque menaçant la vie de tel ou tel ne laisse indifférent aucun membre du groupe qui comprend que ce pourrait être son tour demain ou après-demain.

Supposer l'existence quasi-universelle d'une telle économie de sentiments moraux à l'échelle de tout groupe humain constitue certes une hypothèse très forte. Elle consiste à soutenir qu'au fondement de l'organisation des activités 'anthroponomiques' de production de la vie, et parmi elles les activités de care, il se trouve quelque chose de cet ordre.

Michel Foucault a tenté de le penser à partir de l'entrée 'Pouvoir', ce qui l'a conduit à écrire quelques pages sur la biopolitique qui ont fait couler beaucoup d'encre. Carol Gilligan a identifié la présence spontanée d'une éthique du care chez nombre de préadolescentes américaines, et la façon dont elle a cherché à généraliser cette découverte empirique a elle aussi suscité beaucoup d'intérêt (dans les paragraphes précédents nous avons cependant dégagé de possibles causes sociales de cette éthique). Tout au long du présent article c'est une autre entrée encore que nous avons privilégiée: les formes alternatives d'organisation des activités de care et plus généralement, de production de la vie.

Nous ne croyons pas en la possibilité de découvrir, pour cette 'seconde production', l'équivalent de ce que constitue pour l'économie matérielle la gigantesque machinerie auto-régulante 'du marché'; ou mieux, des marchés. Comme l'a fort bien montré Karl Polanyi, pour faire exister une économie de marché constituée de rapports purement économiques, donc autonomes vis-à-vis des autres types de rapports sociaux, il a d'abord fallu les en arracher. Au demeurant cet arrachement, ce disembedment violent n'est jamais complètement réussi; l'économique, encore aujourd'hui, reste fortement imbriqué dans le politique, voire le culturel.

S'agissant maintenant des rapports de production anthroponomiques, leur 'grande transformation' par autonomisation complète vis-à-vis des autres rapports sociaux paraît inimaginable. Tout au moins ne parvenons-nous pas à imaginer comment ces rapports, parce qu'ils concernent des activités de production de la vie et des chances de vie de tous les membres d'une société donnée, d'une société se pensant comme société et dont les membres sont donc porteurs de sentiments de solidarité mutuelle, pourraient se détacher de son économie morale, de son âme politique, et du modèle culturel commun qui la fait se tenir ensemble. 


\section{Bibliographie}

Bertaux Daniel (1977), Destins personnels et structure de classe. Pour une critique de l'anthroponomie politique, Paris, Presses Universitaires de France.

Bertaux Daniel (1994), "The Anthroponomic Revolution: First Sketch of a Worldwide Process", The Annals of the International Institute of Sociology (New York), IV, p. 177-92.

Bertaux Daniel (2010), Le récit de vie, Armand Colin, $3^{\mathrm{e}}$ ed.

Deleuze Gilles et Félix Guattari (1972), L'AntiEdipe. Capitalisme et schizophrénie. Paris, Éditions de Minuit.

Foucault Michel (1976), Histoire de la sexualité. Vol 1: La volonté de savoir, Paris, Gallimard.

Gilligan Carol (1977), In a different voice: Women's conceptions of self and of morality. Harvard Educational Review, 47, p. 481- 517.

Gilligan, Carol (1982), In a Different Voice. Psychological Theory and Women's Development, Cambridge (USA), Harvard University Press.

Gilligan, Carol (2009), "Making Oneself Vulnerable to Discovery", an interview with Metchild Kiegelmann, Forum Qualitative Research (FQS), vol. 10, $\mathrm{n}^{\circ}$ 2, May.

Hayek, Friedrich von (1944), The Road to Serfdom, Chicago, University of Chicago Press.

Legendre Pierre (1985, ed. augm. 2004), L'Inestimable Objet de la transmission. Étude sur le principe généalogique en Occident, Paris, Fayard.

Mauss Marcel ([1923-1924] 1950) "Essai sur le don. Forme et raison de l'échange dans les sociétés archaïques" p. 145-279 dans Marcel Mauss, Sociologie et anthropologie, Paris, Presses Universitaires de France. Préface de Claude Lévi-Strauss.

Nakano Glenn Evelyn (2010), Forced to Care: Coercion and Caregiving in America, Cambridge, Massachusetts: Harvard University Press.

Nicole-Drancourt Chantal (1989), «Stratégies professionnelles et organisation des familles ", Revue Française de Sociologie, XXX-1, p. 57-80.

Ochiai Emiko (2009), "Care Diamonds and Welfare Regimes in East and South-East Asian Societies: Bridging Family and Welfare Sociology", International Journal of Japanese Sociology, 18-1, p. 60-78.

Piketty Thomas (2913), Le Capital au XXI e siècle, Paris, Éditions du Seuil.

Razavi Sharah (2007), The Political and Social Economy of Care in a Development Context. Conceptual Issues, Research Questions and Policy Options, Gender and Development Program Paper Number 3, UNRISD, Genève.

Sartre, Jean-Paul (1971-1972), L'idiot de la famille: Gustave Flaubert de 1821 à 1857. Paris: Gallimard, 3 tomes.

De Swaan Abram ([1988] 1995), Sous l'aile protectrice de l'État. Paris, Presses Universitaires de France.
Weber Eugen (1976), Peasants Into Frenchmen: The Modernization of Rural France, 1870-1914 (trad. fr. La Fin des terroirs: la modernisation de la France rurale, 1870-1914, Fayard, 1983),

\section{Notes}

1. Ainsi pour la cuisine: s'agit-il sociologiquement de la même activité selon qu'elle est faite à la maison par soi-même, par 'la cuisinière', dans un petit restaurant, dans une chaîne de fast food, dans la cantine d'une école?

2. D'une grande importance analytique, ce point a été souligné par Shahra Razavi, de l'UNRISD en 2007. Prolongeant le courant de pensée ouvert par Esping-Andersen sur les divers types d'arrangements institutionnels fournissant du 'Welfare', et les avancées de Evers ('Welfare triangle') puis Jenson en ce qui concerne le care, Razavi a proposé de distinguer quatre grands 'types' de configuration de rapports sociostructurels. Elle a baptisé sa typologie care diamond, le 'diamant' c'est-à-dire le losange - du care. Nous y reviendrons.

3. Dans l'Introduction de ce numéro de la Revue des Sciences Sociales ont été brièvement rappelées les circonstances atypiques dans lesquelles est née la pensée du care.

4. L'anomalie signalée plus haut, à savoir la résistance passive de l'objet care à se laisser découper et penser analytiquement en fonction des types de rapports de production, se comprend mieux quand on réalise qu'il fait partie - sous toutes ses formes - d'une seconde production dont le corps géant, qui s'est considérablement élargi et transformé au cours des sept dernières décennies, affleure désormais sous forme d'intuitions récurrentes - juste sous la surface de la pensée collective. Mais celle-ci reste encore trop profondément colonisée par la culture de l'argent et sa 'pensée unique' (ultralibérale) pour parvenir à conceptualiser une économie de la production matérielle de la vie qu'elle côtoie pourtant quotidiennement.

5. La 'forme publique' - sans but lucratif est celle qui finance et organise en France la totalité de l'enseignement public (l'Éducation dite Nationale). C'est aussi elle, par l'entremise de la Sécurité Sociale, qui finance l'essentiel des activités de soins médicaux; et qui les organise en partie, tout en maintenant une médecine libérale.

6. La part des soins médicaux dans le Produit Intérieur Brut (PIB) avoisine 11\% dans les pays d'Europe de l'Ouest, au Canada et au Japon; mais $18 \%$ aux USA, non pas parce que les soins et surtout l'accès aux soins y seraient meilleurs (ce serait plutôt le contraire selon tous les observateurs de bonne foi) mais parce qu'ils y sont organisés selon la logique du profit. Les activités d'enseignement constituent en moyenne entre 6 et $8 \%$ du PIB.

7. Ce n'est pas un hasard si, cherchant à construire un Indice de Développement Humain (l'IDH) qui ferait pendant au 'revenu par tête d'habitant' dans les comparaisons internationales, les économistes Mahbub ul Haq et Amartya Sen ont donné un poids majeur au niveau d'éducation (des filles notamment) et à l'espérance de vie (un indicateur synthétique de l'état général de santé d'une population). L'IDH, mesure phare du Programme des Nations Unies sur le Développement, est à l'heure actuelle le meilleur indice de développement anthroponomique d'une société donnée.

8. Nous entendons par là des rapports de pouvoir déjà institués. Le concept de rapports sociaux englobe à la fois les rapports sociostructurels institués par 'le Pouvoir' (au sens de Foucault), les actions du 'Pouvoir' et les résistances à ces rapports de domination: les structures, les actions, et les interactions des acteurs. D'après Foucault (1976), là où il y a domination il y a toujours aussi résistance. "Toujours potentiellement" serait moins optimiste mais sans doute plus exact.

9. Certaines théorisations du care, parce qu'elles se concentrent sur les contenus des activités de care plus que sur les rapports sociostructurels au sein desquels elles s'effectuent, considèrent que par exemple la cuisinière, la servante ou la bonne d'une famille très aisée font du care pour tous les membres de cette famille. Or si les jeunes enfants en sont effectivement dépendants, ce n'est pas le cas de leurs parents qui pourraient fort bien se faire à manger eux-mêmes: pour eux il ne s'agit donc pas de care mais de production anthroponomique.

10. Les infirmières sont souvent considérées comme des auxiliaires des médecins. Mais, du moins en France, leur mouvement collectif revendique une autre conception de la division du travail entre ces deux catégories: "le médecin est le spécialiste de la maladie, disent-elles; mais c'est l'infirmière qui est la spécialiste du malade». L'infirmière, et non le médecin qui est déjà passé à un autre patient. Schématiquement, à l'hôpital les médecins travailleraient principalement sur les corps malades; et les infirmières, avec les personnes hospitalisées. Ce n'est pas tout à fait le même travail. 
11. La forme associative (sans but lucratif, non profit) ne constitue en général qu'une partie négligeable des modes de garde. Elle se développe cependant en tant que forme de gestion de la forme à financement public. Ainsi en Allemagne les Églises (protestantes et catholique), financées par l'impôt cultuel et d'importantes subventions directes, emploientelles 900000 personnes dans leurs écoles et institutions caritatives.

12. La forme publique doit être absolument distinguée de la forme étatique. Il s'agit de deux ensembles distincts mais intersécants. Dans les pays dits 'communistes' (URSS, Chine communiste, Cuba, etc.), c'est-à-dire intégralement soumis au pouvoir d'un "Parti-Etat-Economie" comme disait Moshe Lewin, si les espaces privés étaient très réduits, l'espace public au sens d'Habermasl'était plus encore: car un tel espace est comme une agora, un lieu où les citoyens peuvent librement s'exprimer et échanger informations, points de vue, et débattre sur les politiques à mettre en œuvre; donc, parler politique. Or dans ces régimes l'espace collectif était à peu près entièrement approprié et contrôlé par l'omniprésent Parti-État, le 'Pouvoir'. Une forme étatique sans démocratie, donc non-publique. Car ce que nous appelons ici la forme publique suppose le caractère public des informations et des points de vue, et la possibilité d'une éventuelle participation des usagers / citoyens à son organisation.

13. Certes les ultra-libéraux admettent que le capitalisme engendre des 'inégalités' croissantes: comment nier l'évidence, surtout après le remarquable travail de Piketty (2013)? Mais d'ailleurs, pensentils, pourquoi la nier... si ce n'est au prisme d'un idéalisme égalitaire 'parfaitement utopique'? Le monde des affaires est-il immunisé contre la tentation d'un ordre sociétal stratifié une fois pour toutes en castes, comme dans la dystopie imaginée par Aldous Huxley (Brave New World, 1932)? Gageons que certaines des fondations qu'il finance si généreusement travaillent déjà à ce projet...

14. Pour ce qui est du childcare, l'affaire semble entendue. Il existe en effet un pays qui propose aux mères de jeunes enfants de prendre gratuitement ceux-ci en charge dès l'âge de trois ans, voire deux ans, par un personnel qualifié et dans des bâtiments exclusivement construits à cet effet, pour toute la journée (en semaine seulement). Ce n'est nullement obligatoire, et aucune publicité n'est faite; pourtant $94 \%$ des mères de famille lui confient leur jeune enfant. Ce pays c'est la France, qui y consacre 13 milliards d'euros $(0,6 \%$ de son PIB) et le système est celui des écoles maternelles. À noter que l'abondante littérature anglo-saxonne sur le care, d'inspiration spontanément libérale (mais sans en avoir conscience), passe ce phénomène massif sous silence; soit par idéologie, soit plus vraisemblablement par simple ignorance.

15. Le système fonctionne donc sur la base d'une combinaison, d'un mix de forme for-profit, dominante, et de forme publique. C'est grâce à la forme marchande qu'il doit la très grande diversité de son offre, et la stimulation permanente par la concurrence. On retrouve ces deux avantages dans le mix expérimenté en Suède depuis vingt ans: les friskol. Ce sont des écoles primaires et secondaires entièrement privées, mais gratuites d'accès pour tous car financées par l'État en proportion du nombre d'élèves que leurs parents y ont inscrits de préférence à une école publique. L'enthousiasme initial de nombreux parents d'élèves est quelque peu retombé à la suite d'une baisse générale du niveau - mesuré par PISA- et de la tendance de certains managers à privilégier exagérément le profit au détriment de la qualité des enseignements.

16. Au demeurant l'économie de marché semble impuissante à mettre à la portée de tous les ménages l'une des 'choses' matérielles les plus nécessaires à un mode de vie équilibré: le logement. D'innombrables exemples fournis par l'Histoire du prolétariat anglais de la Révolution industrielle aux immenses bidonvilles et innombrables sans-domicile des métropoles contemporaines du Grand Sud, montre qu'en tant que marchandise pure un logement décent reste inaccessible à une partie importante de la population. D'où l'apparition, partout, de politiques publiques de construction de logements 'sociaux' et d'aides au logement.

17. Il est tout à fait possible que dans de nombreux cas ce soit une forme mixte qui donne les meilleurs résultats. Notre propos ici n'est pas de promouvoir à tout prix la forme publique, mais seulement de montrer que pour ce qui est de la production de la vie, sa pertinence et ses bonnes performances paraissent établies. C'est là une idée qui ne va pas de soi, même en France mais surtout partout ailleurs dans un monde globalisé par l'impact de forces et de politiques ultralibérales. 
